\title{
O papel do enfermeiro na prevenção do câncer no colo de útero
}

\author{
The role of the nurses in the prevention of uteros cancer \\ El papel de las enfermeras en la prevención del cáncer uterino
}

Recebido: 24/09/2021 | Revisado: 30/09/2021 | Aceito: 05/10/2021 | Publicado: 09/10/2021

\author{
Daniele Andrade de Souza \\ ORCID: https://orcid.org/0000-0002-4197-9126 \\ Faculdade Cristo Rei, Brasil \\ E-mail: daniandradedesouza@outlook.com \\ Marli de Oliveira Costa \\ ORCID: https://orcid.org/0000-0002-9616-4477 \\ Faculdade Cristo Rei, Brasil \\ E-mail: marli@faccrei.edu.br
}

\begin{abstract}
Resumo
O presente trabalho tem por objetivo dispor sobre o papel do enfermeiro na prevenção do câncer no colo do útero, desde os exames de prevenção, rastreamento precoce e tratamento. Assim, se faz necessário uma explanação sobre a patologia, bem como sua incidência nas mulheres atualmente, para que o enfermeiro possa estar atualizado a fim de promover um tratamento digno à paciente. Insta ressaltar que o enfermeiro é a peça chave para obter um bom resultado no desenvolvimento do tratamento, apoiando emocionalmente a mulher com a patologia, auxiliando nos exames pertinentes e oferecendo suporte profissional. A metodologia utilizada para apresentar o tema foi a revisão bibliográfica, onde foram pesquisados livros, dissertações, artigos científicos, teses e revistas, através da busca junto ao Google acadêmico, Biblioteca Virtual em Saúde (BVS) e na SciELO, relacionados ao câncer de colo de útero, suas causas, patogenia, possíveis tratamentos e atuação da enfermagem, publicados de 2008 até 2021, sendo utilizado o método dedutivo. O estudo foi dividido em dois capítulos, de modo a ser explicado sobre a importância do enfermeiro em relação ao câncer de colo de útero desde sua prevenção, rastreamento precoce e tratamento, passando pelas necessidades de acolhimento das mulheres acometidas e, por fim, dispondo sobre a ligação da saúde da mulher a prevenção da patologia.
\end{abstract}

Palavras-chave: Câncer de colo de útero; Enfermagem; Prevenção.

\begin{abstract}
The present work aims to discuss the role of nurses in the prevention of uterus cancer, from preventive examinations, early screening and treatment. Thus, an explanation about the pathology is necessary, as well as its incidence in women today, so that the nurse can be updated in order to promote a dignified treatment for the patient. It is worth emphasizing that the nurse is the key to obtaining a good result in the development of the treatment, emotionally supporting the woman with the pathology, assisting in the relevant exams and offering professional support. The methodology used to present the theme was a bibliographic review, where books, dissertations, scientific articles, theses and magazines were searched, through the academic Google search,Virtual Health Library and SciELO, related to uteros cancer, its causes, pathogenesis, possible treatments and performance of nursing, published from 2008 to 2021, using the deductive method. The study was divided into two chapters, in order to be explained about the importance of nurses in relation to cervical cancer from its prevention, early screening and treatment, through the care needs of affected women and, finally, providing about the link between women's health and disease prevention.
\end{abstract}

Keywords: Uterus cancer; Nursing; Prevention.

\section{Resumen}

El presente trabajo tiene como objetivo discutir el papel de las enfermeras en la prevención del cáncer de cuello uterino, desde los exámenes preventivos, la detección precoz y el tratamiento. Por tanto, es necesaria una explicación sobre la patología, así como su incidencia en las mujeres de hoy, para que la enfermera pueda estar actualizada con el fin de promover un trato digno para la paciente. Es de destacar que la enfermera es la clave para obtener un buen resultado en el desarrollo del tratamiento, apoyando emocionalmente a la mujer con la patología, asistiendo en los exámenes pertinentes y ofreciendo apoyo profesional. La metodología utilizada para presentar el tema fue la revisión bibliográfica, donde se realizaron búsquedas de libros, disertaciones, artículos científicos, tesis y revistas, mediante una búsqueda con académicos Google, Biblioteca Virtual en Salud (BVS) y SciELO, relacionados con el cáncer cervicouterino de útero, su causas, patogenia, posibles tratamientos y desempeño de enfermería, publicado de 2008 a 2021, utilizando el método deductivo. El estudio se dividió en dos capítulos, con el fin de explicar la importancia del enfermero en relación al cáncer cervicouterino desde su prevención, tamizaje precoz y tratamiento, pasando por las 
necesidades de atención de las mujeres afectadas y, finalmente, dar a conocer el vínculo entre la salud de la mujer. y prevención de enfermedades.

Palabras clave: Cáncer de cuello uterino; Enfermaría; Prevencíon.

\section{Introdução}

O presente trabalho visa realizar uma revisão bibliográfica em relação ao câncer de colo de útero e a atuação do enfermeiro na sua prevenção, diagnostico e tratamento. O propósito deste artigo é demonstrar a importância que o enfermeiro possui em todo processo do câncer de colo de útero e suas atribuições desde a prevenção até o momento da doença já instalada.

O útero é o órgão interno do aparelho reprodutor feminino, tem um formato similar a uma pêra, sua função é fornecer um ambiente seguro e nutritivo para formação de um bebe durante seu período gestacional, é um abrigo para uma nova vida que nascerá (Ricci, 2008).

O câncer uma palavra que vem do grego karkínos, o que significa caranguejo, atualmente se refere a um conjunto de mais de 100 doenças caracterizados por crescimento celular desordenado (Sabotta, 2012).

De acordo com WHO (2020), o câncer de colo de útero é considerado um problema de saúde publica mundial, vez que há incidência de 530 mil novos casos por ano e 256 mil óbitos em sua decorrência. No Brasil, é a terceira neoplasia mais incidente em mulheres, ficando atrás apenas do câncer de pele não melanoma, sendo um risco estimado de 17,11 casos a cada 100 mil mulheres (Inca, 2019).

Segundo Taquary et al. (2017), o câncer de colo de útero é caracterizado por uma multiplicação desordenada das células localizadas na porção inferior do útero, sendo capaz de atingir tecidos próximos e distantes.

Como sintomas, as mulheres podem apresentar sangramento vaginal durante relações sexuais, corrimento de cor escura com mau cheiro, sendo nos estágios mais avançados pode causar hemorragia, obstrução das vias intestinas e urinarias, contudo, na maioria dos casos, trata-se de uma doença assintomática (Gismondi, et al., 2020).

De acordo com Silva (2013) o enfermeiro deve identificar as necessidades da mulher, de acordo o primeiro contato aos sinais e sintomas, independentemente de suas dificuldades em relação à falta de conhecimento, tabu da sexualidade, deve sempre promover a saúde e prevenção as doenças femininas.

De tal modo, o enfermeiro atua como educador, exercendo sua influência através da comunicação, ele constitui um vínculo com a paciente fazendo com que ela possa expor seus problemas e estilo de vida, onde o enfermeiro consiga identificar qual atendimento a paciente necessita (Silva et al., 2013).

O profissional de enfermagem deve passar muita segurança a paciente, no momento da realização do exame a paciente deve se sentir segura e ao mesmo tempo compreender a importando da realização dos exames de prevenção. $\mathrm{O}$ enfermeiro deve criar um ambiente de acolhimento e privacidade, a fim de fazer um ótimo rastreamento de doenças (Cruz; Loureiro, 2008).

A orientação sobre a prevenção do câncer do colo uterino o enfermeiro pode fazer atração de palestras coletivas ou individuais, com isso as mulheres possam entender o propósito e importância dos exames de prevenção e tomam a iniciativas em fazer esses exames (Silva et al., 2013).

Uma das funções do enfermeiro é relatar a paciente todas as informações sobre os exames preventivos, através de uma boa comunicação, explicando todo procedimentos com muita paciência e acolhimento para conquistar um universo cultural feminino (Eduardo et al., 2008). Insta ressaltar que existem diversos tipos de exames capazes de identificar a doença, como colposcopia, cervicografia, teste de DNA, teste do vírus HPV e o Papanicolau (Oliveira, 2010).

Segundo Oliveira (2010) o enfermeiro pode relatar a paciente a importância do exame citopatológico, realizado de forma correta, pois é realizado nas práticas preventivas no dia a dia feminino, assim podendo ser diagnostica uma doença precoce. 
O profissional de enfermagem deve estar psicologicamente e emocionalmente bem, pois em cada caso atua com sentimentos muito fortes da paciente, sendo que tal fato não pode o abalar. O vínculo entre o enfermeiro e a paciente é muito importante o de demonstra um teor totalmente ético, onde esse é responsável por esclarecer todos os tipos informações e as manter em sigilo (Moura et al., 2010).

Quando muitas mulheres procuram fazer os exames de prevenção ou até mesmo o tratamento da doença, é sinal que o trabalho do enfermeiro, juntamente com a sua equipe, está sendo muito bem feito, demonstrando a importância da prevenção para o câncer no colo uterino (Soares et al,2011).

Outra importante atuação do enfermeiro a prevenção e detecção precoce do câncer de colo uterino é a realização de busca ativa, pois algumas mulheres não fazem os exames preventivos e outras abandonam o tratamento. Cabe ao enfermeiro buscar essas mulheres orientá-las e encaminhar para ter uma oportunidade de se cuidar (Oliveira, et al., 2010).

O enfermeiro deve também orientar as mulheres a ter as medidas de auto-cuidados e incentivar a realizar essas medidas, isso é com certeza uma prevenção do câncer do colo uterino, a eficiente da equipe de enfermagem traz mais confiança, assim diminuindo a incidência de casos de câncer no colo uterino.

É bem claro que o enfermeiro é o principal responsável a atenção primária e se torna capaz de analisar as dificuldades encontradas para a realização dos exames de prevenção, com intuito de buscar soluções adequadas, com uma postura crítica e reflexiva para uma assistência mais humanizada ao grupo feminino.

\section{Metodologia}

A presente pesquisa qualitativa fora realizada de forma descritiva, através de revisão de literatura nas bases de dados da Biblioteca Virtual em Saúde (BVS), Google Acadêmico e SciELO (Eletronic Library Online) (Pereira, et al., 2019)

Tendo em vista o tema proposto, foram considerados monografias, dissertações, artigos científicos e teses com pesquisas quantitativas e qualitativas que abordassem de maneira clara o papel do enfermeiro na prevenção do câncer de colo de útero, publicados de 2008 à 2020, em português.

A fim de proceder a pesquisa nas bases de dados anteriormente mencionadas, utilizou-se as seguintes palavras chaves: atuação do enfermeiro no câncer de colo de útero, câncer de colo de útero, papanicolau.

Após, o material encontrado foi submetido a dois momentos classificatórios, sendo que no primeiro os artigos foram selecionados considerando o título, resumo e data de publicação. Posteriormente, esses foram classificados de acordo com a sua relevância ao tema investigado, após leitura minuciosa.

Insta salientar que houve maior facilidade e interesse em busca de artigos junto ao Google Acadêmico, vez que em tal plataforma há um compilado maior de informações, pois engloba as demais plataformas anteriormente mencionadas.

Assim, superado o momento classificatório, os artigos selecionados foram separados de modo que a organização do presente estudo se desse de uma maneira sistemática, fora confeccionada a tabela a seguir para melhor visualização do material encontado: 
Tabela 1. Buscas nas bases de dados e categorização do material levantado.

\begin{tabular}{cccc}
\hline Base de dados & Publicações obtidas & Publicações excluídas & $\begin{array}{c}\text { Publicações } \\
\text { selecionadas }\end{array}$ \\
\hline BVS & 218 & 215 & 3 \\
\hline Google Acadêmico & 12.100 & 12.090 & 8 \\
\hline SciELO & 4 & 3 & 1 \\
\hline Total & 12.322 & 12.310 & 12 \\
\hline
\end{tabular}

Fonte: Autores (2021).

Assim, torna-se possível denotar que existe uma vasta quantidade de material que trata sobre o câncer de colo de útero, sendo que foram selecionados àqueles que mais se encaixavam no tema proposto a fim de que não extrapolasse a delimitação do presente trabalho.

\section{O Câncer de Colo de Útero e Seu Contexto na Saúde Pública}

O câncer de colo de útero $(\mathrm{CCU})$ é atualmente a segunda neoplasia mais comum em mulheres no mundo, estando em terceiro no Brasil, causando mais de 200 mil óbitos por ano. A taxa de incidência de CCU ocupa a primeira posição na região norte do país e segunda nas regiões Centro-Oeste e Nordetes. No Sul e Sudeste, o CCU é o terceiro e quarto tipo de câncer mais incidente, respectivamente (Ministério da Saúde, 2016).

De acordo com Cruz e Loreiro (2008), há uma alta incidência de câncer cervico-uterino em mulheres na faixa etária de 20 a 29 anos, aumentando-se o risco em mulheres de 45 a 49 anos de idade. No que tange à demografia, os países que se encontram em desenvolvimento possuem mais novos casos no percentual de 80\%. Cabe ressaltar que nesses países, o diagnostico é tardio, sendo a sobrevida média cinco anos menores em relação aos países desenvolvidos (INCA, 2005).

Para Lopes e Ribeiro (2017), "Este câncer é causado, majoritariamente, por infecção persistente via subtipos oncogênicos do Papilomavírus Humano (HPV), transmitido sexualmente, sendo esta infecção responsável por cerca de $70 \%$ dos cânceres cervicais".

Da família papilomaviridae, os HPV's são capazes de provocar lesões na mucosa ou na pele. Atualmente são conhecidos mais de 200 tipos de HPV, sendo classificados de baixo risco de câncer e de alto risco de câncer, sendo que somente os últimos estão diretamente relacionamentos a tumores malignos (Brasil, 2010).

O controle do câncer de colo de útero na saúde publica e privada é norteado pelos parâmetros e diretrizes do Sistema Único de Saúde - SUS, de modo articulado compondo uma atenção à saúde primária na perspectiva de integralidade, sendo guiado por uma linha de cuidado, responsável pelo fluxo assistencial e acompanhamento clínico da evolução da enfermidade (Lopes; Ribeiro, 2017).

À atenção básica e à atenção especializada - média e alta complexidade - correspondem modalidades de atenção à saúde, sendo: promoção, prevenção, diagnóstico, tratamento, reabilitação e cuidados paliativos. A promoção diz respeito a ações transversais visando promover melhorias na saúde da população, controlar doenças e agravos à saúde, incluindo ações que ampliem a informação e reduzam as dificuldades de acesso a serviços de saúde. (Inca, 2012).

Sobre o acesso à saúde dispõem Travasso e Castro (2012):

Esses estudos analisam - de modo isolado ou articulado - a oferta de serviços de saúde; as características da relação entre procura e utilização dos serviços de saúde, reconhecendo aspectos e/ ou dimensões que atuam como facilitadores ou obstrutores dessa utilização por potenciais usuários; e, os resultados da prestação de serviços de saúde. 
De tal modo, torna-se imprescindível o acolhimento e direcionamento do enfermeiro para que os exames pertinentes sejam realizados na periodicidade recomendada, vez que quando maior o número de mulheres que se submetem aos exames e são diagnosticadas precocemente, maior a chance de cura e sobrevida, acarretando a melhor prestação de serviços por parte da equipe de saúde.

\subsection{Métodos de prevenção, rastreamento e o papanicolau}

Como já mencionado, o CCU é um câncer com mortalidade elevada, que, mesmo com as campanhas e programas governamentais de prevenção, ainda continua sendo um problema de Saúde Pública no Brasil, embora já apresente conhecimentos técnicos de prevenção suficientes para fornecer um dos mais altos potenciais de cura (Loureiro; Cruz, 2008).

Alguns estudos apontam que poucas mulheres possuem conhecimento de fato sobre o CCU, sendo que essa falta de informação é mais freqüente em regiões de menor poder aquisitivo, seguido da baixa escolaridade (Silva, et al., 2020).

Em decorrência da falta de informação, essas mulheres se tornam mais vulneráveis a contrair a doença, bem como ter mais danos por essa causados, vez que a grande maioria terá diagnostico tardio por não conseguir identificar os sinais e sintomas. (Silva, et al., 2020)

A medida de diagnostico mais eficaz e célere se dá por meio de um exame simples chamado citopatológico de colo do útero, popularmente conhecido como Papanicolau. Esse exame é responsável por indicar a presença de leões neoplasticas ou até pré-neoplásticas, a fim se de iniciar o tratamento e interromper sua evolução (Barcelos, 2011). Trata-se de um exame indolor, eficaz e de baixo custo, que deve ser oferecido às mulheres após o início da vida sexual (Ribeiro, et al, 2019).

Em 2009 fora estimada uma redução de 80\% na mortalidade decorrente de CCU por meio de rastreamento de mulheres na faixa etária de 25 aos 65 anos com a submissão dessas ao exame de papanicolau e/ou tratamento de lesões com alto potencial de malignidade ou carcinoma "in situ" (Brasil, 2009).

Cabe ressaltar o que fora descrito por AMBOA et al. (2019) sobre a realização do exame de papanicolau sem saber sobre seu real benefício à saúde, bem como sobre o fato de minimização de fatores de riscos podem atuar como prevenção, sendo eles, início precoce da vida sexual, multiplicidade de parceiros, uso prolongado de contraceptivos orais, tabagismo, higiene intima adequada, entre outros. Recomenda-se que todas as mulheres que tem vida sexual ativa realizem o exame anualmente (Santos, 2013).

No que tange a atuação do enfermeiro, Gozzo et al. 2010, alega que "compete aos profissionais de saúde dar orientações adequadas às mulheres quanto à importância da realização do exame de papanicolau para detecção precoce do câncer de colo de útero independentemente dos fatores de risco e idade."

Ademais, cabe ao enfermeiro fornecer as orientações necessárias à paciente, dispondo sobre os métodos preventivos, identificação de efeitos colaterais, indicação de acompanhamento psicológico em caso de diagnóstico de CCU, bem como a criação de campanhas de coletas de exames e quebras de tabus (Frigato; Lak, 2005).

Sob a mesma perspectiva Carneiro et al. (2019) relacionada algumas ações que podem ser promovidas pelos enfermeiros no combate ao CCU:

Cabe aos enfermeiros por sua proximidade com a população, uma educação em saúde de maneira integral, incentivando as consultas de enfermagem, abordagens para esclarecimento de dúvidas, riscos, sinais e sintomas, pois essas práticas favorecem mudanças de comportamentos e de atitudes das mulheres

De acordo com Carneiro et al. (2019) a prevenção ao CCU pode ocorrer em prevenção primária e secundária, sendo a primeira de baixo custo, pautada na educação em saúde, dispondo sobre os fatores de risco e fortalecendo intervenções como a recente vacina da HPV, disponível na rede pública de saúde para meninos e meninas de até 14 anos. Já a prevenção secundária 
consiste na diminuição da incidência, prevalência e mortalidade com o rastreamento pelo exame para detecção precoce das lesões precursoras.

A fim de garantir a eficácia e eficiência deste rastreamento, o Ministério da Saúde instituiu alguns programas voltados à saúde da mulher, como o Programa de Assistência Integral à Saúde da Mulher, em 1986, o Programa Nacional de Combate ao Câncer do Colo do Útero, em 1997, sendo esses responsáveis pelas diretrizes e promoção do exame cipatológico (Barbosa, et al., 2011).

Assim sendo, de acordo com a realidade em que está inserido, o enfermeiro pode atuar de acordo com o perfil da comunidade, contando com o apoio de agentes públicos, bem como de agentes comunitários de saúde, a fim de promover uma atuação ativa em educação de saúde, aconselhando e reduzindo preconceitos e mitos sobre o exame, promovendo um ambiente adequado para que as mulheres se sintam seguras e possam expressar queixas e dúvidas (Nascimento, 2010; Costa, 2017; Carneiro et al., 2019)

Assevera-se que o enfermeiro é habilitado ao longo da vida acadêmica para realizar a coleta de exame papanicolau, com respaldo na lei do Exercício Profissional 7498/86, além da coleta, possui capacidade técnica para interpretação de resultados, fazer encaminhamentos e monitorar casos suspeitos e confirmados de câncer de colo de útero (Oliveira, 2014).

\subsection{Tratamento}

Sobre o pós diagnostico Mello et al. (2009) descreve que "a vida da mulher toma um caminho diferente do que ela idealizava, pois, essa doença traz mudança em todos os aspectos de sua vida, o tratamento provoca possíveis reações como mutilações, náuseas, vômitos, queda de cabelo e disfunções sexuais"

Após a realização do exame e sendo encontradas lesões precursoras, essas podem ser classificadas de acordo com o seu grau de evolução em neoplasia intraepitelial cervical (NIC), sendo um distúrbio que acomete o epitélio uterino e dividido em três estágios de acordo com a sua proporção. A NIC I acomete somente camadas basiais do epitélio e é considerada de grau leve. Já a NIC II, de grau moderado, avança de três a quatro camadas do epitélio, mas conserva as camadas superficiais. A displasia grave, chamada de NIC III atinge todas as camadas do epitélio do colo uterino, podendo chegar a invadir o tecido conjuntivo, razão pela qual se passa a ter um carcinoma escamoso invasivo (Carneiro, et al. 2019; Oliveira, 2014).

De acordo com Corrêa (2011):

"o tratamento para o câncer de colo uterino dependerá do estadiamento da Federation of Gynecology and Obstretris (FIGO) e Tumor, Linfonodos e Metástase (TNM), com base no exame histológico, idade da mulher, condições clínicas apresentadas, levando em conta o desejo da mulher de reprodução."

Após o diagnostico, inicia-se uma fase conturbada na vida da mulher, dando-se inicio ao tratamento, às vezes longo, causador de efeitos colaterais físicos e mentais, vez que são muitas as preocupações decorrentes do câncer de colo de útero (Melo, 2009).

Devido a tantas inseguranças é de suma importância que a equipe de enfermagem contribua para que a mulher tenha uma melhor qualidade de vida, em aspectos físicos, psicológicos e sociais, sempre levando em consideração os parâmetros da Organização Mundial de Saúde e situando a paciente no seu convívio social e de afeto (Correa, 2011; Santos, 2014; Carneiro et al., 2019).

Ademais, torna-se importante que após o diagnostico toda a família da paciente seja amparada, devendo o enfermeiro a orientar sobre o tratamento, empatia, elencar possíveis alterações, a importância da continuidade do tratamento e do apoio familiar em um momento tão delicado, sempre dando voz à paciente para amenizar medos e angustias (Carneiro et al., 2019). 
Vargas et al. (2013) trata sobre a possibilidade de uso da Sistematização da Assistência de Enfermagem (SAE) como ferramenta de trabalho da equipe de enfermagem, a fim de fornecer assistência integral à paciente, proporcionando a ela um apoio individualizado baseado na identificação de problemas e dando embasamento para possíveis intervenções.

\section{O Papel do Enfermeiro na Prevenção ao CCU}

Considerando a existência de exame de prevenção ao câncer de colo de útero, sendo esse indolor, simples e rápido, fica a cargo do enfermeiro orientar sobre sua importância, bem como proceder ao encaminhamento das mulheres com vida sexual ativa para a sua realização. Cabe mencionar que mesmo com toda a facilidade anteriormente mencionada, bem como o fato do exame ser disponibilizado pelo SUS, a cobertura ainda não é considerada satisfatória, havendo barreiras a serem rompidas pelos profissionais da saúde para que a prevenção ocorra no tempo hábil.

Insta ressaltar que as coletas citológicas são realizadas, prioritariamente, por enfermeiros em nível de atenção primária à Saúde, os quais são responsáveis pelo cuidado preventivo ao câncer de colo de útero, desenvolvendo estratégias para que mais mulheres tenham conhecimento sobre a gravidade da patologia, bem como sobre a importância de sua prevenção (Alves, et al. 2021). Sobre o tema Oliveira e Fernandes (2017) discorrem sobre a necessidade de que os enfermeiros realizem campanhas para coleta de papanicolau após os horários habituais, a fim de que o maior número possível de mulheres se submetam ao exame.

Após, de acordo com Conceição et al. (2017), se faz necessário o redirecionamento de mulheres com alterações constatadas no papanicolau para consulta ginecológica, a fim de que seja realizado um atendimento de qualidade, bem como ocorra o diagnostico precoce da doença.

Nas Unidades Básicas de Atendimento - UPA, é papel do enfermeiro exercer atividades técnicas de sua competência, administrativas e educacionais com as pacientes a fim de dirimir tabus, preconceitos e buscar disseminar as vantagens da prevenção primária a neoplasia. Assim, deve ajudar a direcionar as ações da enfermagem para a resolução ou minimização das necessidades individuais dos pacientes.

Em relação ao combate ao câncer, a atividade dos enfermeiros é grande valia, atuando em diversas áreas, como: consultas de enfermagem, ações educativas, contato para provimento de recursos materiais e técnicos, investigação, comunicação de resultados, encaminhamento para consultas médicas e afins. Atuando através de tais medidas para a prestação de um serviço de saúde eficaz e eficiente para que o diagnóstico da patologia ocorra o mais breve possível, focando nas chances de cura. (Leite et al., 2020).

Segundo Ramos et al. (2014), o desenvolvimento de ações relacionadas ao câncer de colo de útero, ultrapassa as questões de saúde básica, de modo que deve o enfermeiro ter conhecimento e dispor sobre os riscos da doença, bem como tentar proporcionar uma assistência de qualidade:

Assim, para o desenvolvimento de ações efetivas no controle do CCU, é importante o envolvimento do enfermeiro com os outros profissionais da ESF a fim de utilizarem os conhecimentos sobre a epidemiologia, fatores de risco, sinais, sintomas e instrumentos existentes para a prevenção do referido câncer. É preciso, ainda, que haja uma real preocupação com a gravidade dessa doença por parte de todos os responsáveis nos níveis federal, estadual e municipal visando garantir o acesso aos serviços de saúde, uma assistência de qualidade proporcionada por profissionais qualificados e dentro de uma infraestrutura adequada

A busca ativa é uma estratégia de grande relevância nas ações preventivas, sendo que essa é realizada pelos agentes comunitários de saúde - ACS, em conjunto com enfermeiros. Assim, após o fornecimento de listas de mulheres em idade passível de realização de citopatológico pelos ACS, em parceria, devem ser realizados projetos para que as mulheres que não comparecem aos unidades básicas de saúde realizem o exame preventivo regularmente (Alves, et al. 2021). 
Para Silva et al. (2019), o enfermeiro tem uma grande ligação com o ACS, podendo realizar conjuntamente os esclarecimentos para a população como também promoverem campanhas para incentivarem as mulheres à realização dos exames.

É de extrema importância e necessidade que haja a disseminação de informações sobre o papanicolau pelo enfermeiro responsável pela coleta, bem como argumenta Farias (2019):

O enfermeiro pode e deve esclarecer sobre a importância do exame durante as rodas de conversa realizadas com as mulheres na UBS, onde a realidade é problematizada por meio da conversação, de forma que a conscientização possa ocorrer. As rodas de conversa possibilitam encontros dialógicos criando assim possibilidades de produção e ressignificação de saberes e sentidos sobre a experiência de cada participante, produzindo conhecimentos coletivos e contextualizados a partir da fala crítica e da escuta sensível, favorecendo o entrosamento e a confiança entre os participantes

Infelizmente, a saúde intima feminina ainda é um tabu na sociedade brasileira, de modo que quanto mais a conversa se der de forma leve e descontraída, melhor será a aceitação de realização do exame preventivo, sendo uma forma de conscientização e promoção de autoconhecimento capaz de desenvolver confiança entre as participantes. De tal modo, a educação em saúde, por meio de esclarecimento de dúvidas e medos abre horizontes para a redução da mortalidade do CCU (Medeiros et al., 2021).

Barbosa et al. (2020) e Silva et al. (2019) destacam que dentre as razões que levam a não realização de exame preventivo, se destacam a vergonha, medo, pudor e preconceito, vez que para muitas, trata-se de um exame invasivo, em uma região intima que só poderia ser visitada pelo companheiro, tornando-se de extrema importância que os profissionais de saúde procurem meios para minimizar tais sentimentos durante a realização do exame, sempre dispondo sobre sua importância para que a paciente se sinta segura e acolhida e retorne anualmente para a coleta.

Ainda, é necessário que o enfermeiro adentre a realidade daquela paciente, seja socioeconomicamente, seja culturalmente, para que essa se sinta respeitada a fim de não procurar tratamento somente quando a doença já estiver avançada, como elenca Leite et al. (2020):

O enfermeiro precisa trabalhar de forma ética para respeitar estigmas relacionados a valores morais, religiosos e culturais, abrindo-se espaço para um olhar holístico, respondendo a questões que impactam a sexualidade, a saúde e a adesão ao exame preventivo, além disso, o profissional de enfermagem deve ver a mulher como um ser integral e educá-la no sentido de desenvolver um comportamento preventivo, buscando a Unidade mesmo na ausência de sintomas

Devido às dificuldades culturais, sociais, desinformação e tabus, a adesão ao exame preventivo no Brasil é considerada baixa, vez que milhões de mulheres em idade adequada nunca realizaram papanicolau. E, ainda, das que realizam, cerca de $40 \%$ não voltam para buscar o resultado, tornando-se novamente de extrema importância o relacionamento da enfermagem com os ACS para que aquelas que possuem alterações diagnosticadas na coleta procurem o tratamento médico o mais rápido possível (Dantas et al., 2018).

Assim sendo, a busca ativa realizada pelos ACS, sob supervisão de um enfermeiro, torna-se peça fundamental na prevenção do $\mathrm{CCU}$, estando esta estratégia diretamente relacionada à resultados positivos. Sendo imprescindível a realização de exame papanicolau ofertado pelo Ministério da Saúde em todas as mulheres com vida sexual ativa, cabendo ao enfermeiro possuir competência técnica e cientifica para realizar o exame com excelência a fim de identificar as lesões precursoras do câncer de colo de útero (Medeiros et al. 2021).

$\mathrm{O}$ controle do câncer de colo de útero está diretamente interligado à promoção de saúde, prevenção da patologia e qualidade de vida. Assim, ao adentrar em tais áreas, o enfermeiro passa a desempenhar atividades desde visitas domiciliares à 
prestação de enfermagem humanizada, cooperando para que haja o melhor atendimento às mulheres, sempre as ensinando sobre cada etapa do exame de coleta, bem como explanando sobre a importância de realização do exame e do diagnostico precoce (Leite et al., 2019).

O conglomerado de fatores que levam ao diagnostico do câncer de colo de útero tardio é ligado a políticas de prevenção deficitárias, de modo que é imperativo que os enfermeiros, bem como toda a equipe de saúde, tenham um olhar para a realidade do local em que laboram, para que sejam vencidas as barreiras para realização de exame preventivo, bem como que seja dado ouvido as experiências das mulheres que não se submeteram ao exame anteriormente (Leite et al., 2019).

O objetivo das estratégias para prevenção e controle do câncer de colo de útero é reduzir sua ocorrência e as conseqüentes repercussões físicas, psiquiátricas e sociais. Assim, considerando que o fato de risco do ambiente é responsável por $80 \%$ a 90\% do CCU, a detecção precoce do HPV e lesões precursoras podem impedir a progressão do câncer. (Leite, et al., 2019).

Por conseguinte, resta claro que o enfermeiro precisa trabalhar de maneira estritamente profissional, honrando seu juramento, a ética, respeito a individualidade de cada paciente, a fim de abrir novos horizontes para as mulheres que não possuem conhecimento a cerca do tema e estar apto a responder toda e qualquer dúvida que a paciente tenha a ter relacionado ao exame papanicolau e ao câncer de colo de útero. Ainda, cabe ressaltar que não se trata de um trabalho individual do enfermeiro, e sim envolvendo toda a equipe de saúde, bem como os gestores públicos, vez que o desconhecimento do exame se trata de um reflexo social e cultural que necessita de clareza para ser alterado (Leite et al., 2019).

\section{Resultados e Discussão}

Da pesquisa realizada foram encontradas 12.322 publicações nas bases de dados pesquisadas - Biblioteca Virtual em Saúde (BVS), Google Acadêmico e SciELO (Eletronic Library Online) -, sendo que dessas 12.310 não se enquadraram nos requisitos pré-estabelecidos, chegando-se ao numero de 12 publicações que contribuíram de maneira considerável para a compreensão do papel do enfermeiro na prevenção ao câncer de colo de útero.

Desses, vale destacar Carneiro et al. (2019) que dispôs de maneira clara e de fácil compreensão sobre as atribuições do enfermeiro em relação ao câncer de colo de útero desde sua prevenção até os cuidados pós diagnóstico, argumentando sobre a imprescindibilidade dos conhecimentos técnicos que o profissional deve ter para que todas as duvidas da paciente sejam sanadas e essa possa realizar o exame com segurança.

Diante de tais resultados, a autora elaborou o seguinte quadro para descrever as evidências encontradas na pesquisa, trazendo o número do artigo, base de dados em que foi localizado, ano da publicação e autor, título, objetivo e principais pontos, conforme se vê abaixo:

Quadro 1. Aspectos relevantes do material bibliográfico selecionado.

\begin{tabular}{|c|c|c|c|c|c|}
\hline $\mathrm{N}^{\circ}$ & $\begin{array}{ll}\begin{array}{l}\text { Base } \\
\text { dados }\end{array} & \text { de } \\
\end{array}$ & Título & Autor e ano & Objetivos & Principais pontos \\
\hline 1 & $\begin{array}{l}\text { Google } \\
\text { Acadêmico }\end{array}$ & $\begin{array}{l}\text { Conhecimento de } \\
\text { mulheres sobre } \\
\text { câncer de colo do } \\
\text { útero: Uma revisão } \\
\text { integrativa }\end{array}$ & $\begin{array}{l}\text { SILVA, M. L., } \\
\text { Nunes, J. S. S., } \\
\text { Oliveira, K. S., \& } \\
\text { Leite, T. A. S.. } \\
(2020) .\end{array}$ & $\begin{array}{l}\text { Verificar o conhecimento } \\
\text { de mulheres no climatério } \\
\text { sobre câncer de colo do } \\
\text { útero através de uma } \\
\text { revisão integrativa }\end{array}$ & $\begin{array}{l}\text { Elencar sobre o conhecimento das } \\
\text { mulheres sobre o câncer de colo de } \\
\text { útero, sobre os sinais e sintomas, o } \\
\text { tratamento e a prevenção }\end{array}$ \\
\hline 2 & BVS & $\begin{array}{l}\text { O Papel do enfermeiro } \\
\text { frente ao câncer de colo } \\
\text { uterino }\end{array}$ & $\begin{array}{l}\text { Carneiro, C. P. F., } \\
\text { Pereira, D. M., } \\
\text { Pereira, A. T \& et } \\
\text { al. (2019). }\end{array}$ & $\begin{array}{l}\text { Descrever quais são as } \\
\text { atribuições do enfermeiro } \\
\text { no manejo do câncer de } \\
\text { colo uterino (CCU), desde } \\
\text { sua prevenção até o } \\
\text { momento da doença já } \\
\text { instalada. }\end{array}$ & $\begin{array}{l}\text { Dispôs de maneira clara e de fácil } \\
\text { compreensão sobre as atribuições do } \\
\text { enfermeiro em relação ao câncer de colo } \\
\text { de útero desde sua prevenção até os } \\
\text { cuidados pós diagnóstico, argumentando } \\
\text { sobre a imprescindibilidade dos } \\
\text { conhecimentos técnicos que o }\end{array}$ \\
\hline
\end{tabular}




\begin{tabular}{|c|c|c|c|c|c|}
\hline & & & & & $\begin{array}{l}\text { profissional deve ter para que todas as } \\
\text { duvidas da paciente sejam sanadas e } \\
\text { essa possa realizar o exame com } \\
\text { segurança. }\end{array}$ \\
\hline 3 & $\begin{array}{l}\text { Google } \\
\text { Acadêmico }\end{array}$ & $\begin{array}{l}\text { Atribuições } \\
\text { enfermeiro no } \\
\text { rastreamento do câncer } \\
\text { de colo do útero em } \\
\text { pacientes atendidas na } \\
\text { Unidade Básica de } \\
\text { Saúde }\end{array}$ & $\begin{array}{l}\text { Leite, A. C., Silva, } \\
\text { M. P. B., Alves, R. } \\
\text { S. S., Feitosa, L. } \\
\text { M. H., Ribeiro, R., } \\
\text { Prado, A. M., \& } \\
\text { Soares, N. C. F. B. } \\
\text { (2020). }\end{array}$ & $\begin{array}{l}\text { Analisar as evidências } \\
\text { científicas acerca das } \\
\text { atribuições do enfermeiro } \\
\text { no rastreamento do câncer } \\
\text { de colo do útero em } \\
\text { pacientes atendidas na } \\
\text { Unidade Básica de Saúde }\end{array}$ & $\begin{array}{l}\text { A enfermagem tem um papel } \\
\text { imprescindível na prevenção do câncer } \\
\text { de colo de útero, identificando as } \\
\text { populações de alto risco, desenvolvendo } \\
\text { ações de planejamento, controle e } \\
\text { supervisão de programas de educação e } \\
\text { prevenção, e assim contribuindo para } \\
\text { um diagnóstico precoce da doença. }\end{array}$ \\
\hline 4 & SciELO & $\begin{array}{l}\text { A Comunicação na } \\
\text { Abordagem Preventiva } \\
\text { do Câncer do Colo do } \\
\text { Útero: importância das } \\
\text { influências histórico- } \\
\text { culturais e da } \\
\text { sexualidade feminina } \\
\text { na adesão às } \\
\text { campanhas }\end{array}$ & $\begin{array}{l}\text { Cruz, L. M. B., \& } \\
\text { Loureiro, R. P. } \\
\text { (2008). }\end{array}$ & $\begin{array}{l}\text { Discutir possibilidades } \\
\text { estratégicas que possam ser } \\
\text { eficazes para uma maior } \\
\text { adesão feminina às } \\
\text { campanhas preventivas, foi } \\
\text { considerada a importância } \\
\text { da forma de abordagem } \\
\text { dos profissionais de saúde, } \\
\text { salientando para que atuem } \\
\text { correspondendoras ar aros } \\
\text { valores culturais } \\
\text { apresentados pelas } \\
\text { mulheres. }\end{array}$ & $\begin{array}{l}\text { Ressaltou-se a linguagem utilizada nas } \\
\text { campanhas de prevenção do câncer do } \\
\text { colo do útero, atentando para o fato de } \\
\text { que conceitos transmitidos podem } \\
\text { endossar valores culturais de abordagem } \\
\text { do feminino que dificultam a } \\
\text { identificação e a adesão de muitas } \\
\text { mulheres }\end{array}$ \\
\hline 5 & $\begin{array}{l}\text { Google } \\
\text { Acadêmico }\end{array}$ & $\begin{array}{l}\text { O conhecimento do } \\
\text { enfermeiro sobre a } \\
\text { prevenção do câncer } \\
\text { de colo de útero na } \\
\text { atenção básica }\end{array}$ & 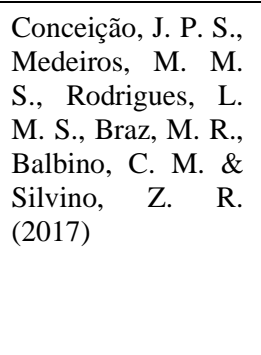 & $\begin{array}{l}\text { Identificar o conhecimento } \\
\text { do enfermeiro da Atenção } \\
\text { Básica quanto à prevenção } \\
\text { do câncer de colo de útero, } \\
\text { descrever a percepção do } \\
\text { enfermeiro quanto à } \\
\text { prevenção deste câncer, e } \\
\text { identificar as atividades de } \\
\text { prevenção realizadas pela } \\
\text { Atenção Básica de Saúde }\end{array}$ & $\begin{array}{l}\text { Foram discutidas cinco categorias sobre } \\
\text { prevenção, educação em saúde, coleta } \\
\text { do exame citopatológico, vacina contra } \\
\text { HPV e ações do planejamento da } \\
\text { prevenção do câncer de colo de útero. }\end{array}$ \\
\hline 6 & BVS & $\begin{array}{l}\text { Alterações } \\
\text { citopatológicas e } \\
\text { fatores de risco para } \\
\text { ocorrência do câncer de } \\
\text { colo uterino }\end{array}$ & 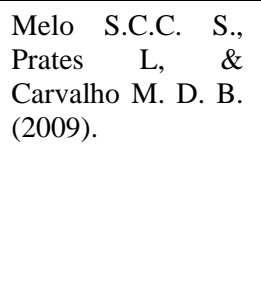 & $\begin{array}{l}\text { Verificar alterações } \\
\text { citopatológicas e fatores de } \\
\text { risco para o câncer de colo } \\
\text { uterino em mulheres } \\
\text { usuárias do Sistema Único } \\
\text { de Saúde de um município } \\
\text { de pequeno porte do norte } \\
\text { do Paraná, de 2001 a } 2006 .\end{array}$ & $\begin{array}{l}\text { Foram entrevistadas } 25 \text { mulheres, a } \\
\text { maioria apresentou algum fator de risco } \\
\text { como: tabagismo, doenças sexualmente } \\
\text { transmissíveis, uso de anticoncepcional } \\
\text { hormonal, número de parceiros, início } \\
\text { precoce da atividade sexual. }\end{array}$ \\
\hline 7 & BVS & $\begin{array}{l}\text { A Atuação do } \\
\text { Enfermeiro de PSF no } \\
\text { Processo da Realização } \\
\text { do Papanicolau }\end{array}$ & $\begin{array}{l}\text { Silva, S. } \quad \text { M. S. } \\
(2013)\end{array}$ & $\begin{array}{l}\text { Avaliar as razões que } \\
\text { levam a não realização do } \\
\text { exame. Investigar o } \\
\text { conhecimento que a mulher } \\
\text { tem sobre a importância do } \\
\text { exame. Avaliar se a } \\
\text { abordagem realizada pelo } \\
\text { profissional é facilitador } \\
\text { para adesão da mulher em } \\
\text { realizar o exame }\end{array}$ & $\begin{array}{l}\text { A ESF traz elevação da qualidade de } \\
\text { vida das mulheres, em virtude da busca } \\
\text { pelo atendimento humanizado } \\
\text { rompimento de barreiras físicas e sociais } \\
\text { por ter presença do profissional de saúde } \\
\text { atuando na comunidade, favorecendo } \\
\text { vínculo, fortalecendo uma melhor } \\
\text { abordagem na busca em mudar a } \\
\text { realidade atual, diminuindo a incidência } \\
\text { da mortalidade feminina por uma doença } \\
\text { de fácil prevenção. }\end{array}$ \\
\hline 8 & $\begin{array}{l}\text { Google } \\
\text { Acadêmico }\end{array}$ & $\begin{array}{l}\text { Saúde da Mulher: } \\
\text { Medidas preventivas } \\
\text { para o câncer de colo } \\
\text { do útero }\end{array}$ & 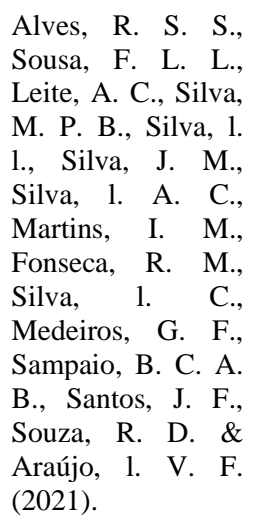 & $\begin{array}{l}\text { Compreender os fatores de } \\
\text { risco para o câncer de colo } \\
\text { do útero, e descrever as } \\
\text { medidas preventivas para } \\
\text { a doença. }\end{array}$ & $\begin{array}{l}\text { Importância da percepção dos } \\
\text { profissionais de saúde atuantes em } \\
\text { unidades de Atenção Primária à Saúde, } \\
\text { frente as orientações quanto as medidas } \\
\text { preventivas para o câncer uterino, } \\
\text { visando promover práticas assistenciais } \\
\text { de acolhimento, e uma percepção } \\
\text { qualificada para atender essa demanda, } \\
\text { viabilizando o acolhimento inicial, } \\
\text { acompanhamento integral, assistencial e } \\
\text { humanizado. }\end{array}$ \\
\hline
\end{tabular}




\begin{tabular}{|c|c|c|c|c|c|}
\hline 9 & $\begin{array}{l}\text { Google } \\
\text { Acadêmico }\end{array}$ & $\begin{array}{l}\text { Citopatologia como } \\
\text { prevenção do câncer do } \\
\text { colo uterino }\end{array}$ & $\begin{array}{l}\text { Siqueira, G. } \quad \text { S. } \\
(2014)\end{array}$ & $\begin{array}{l}\text { Demonstrar a importância } \\
\text { do exame citopatológico } \\
\text { como principal } \\
\text { método diagnóstico do } \\
\text { câncer do colo uterino, } \\
\text { bem como descrever as } \\
\text { principais alteraçóes/lesões } \\
\text { encontradas no colo uterino } \\
\text { e relatar o papel da } \\
\text { enfermagem na prevenção } \\
\text { do câncer do colo uterino }\end{array}$ & $\begin{array}{l}\text { Orientação de enfermagem de qualidade } \\
\text { para as mulheres em idade reprodutiva, } \\
\text { visando à realização da citopatologia } \\
\text { conforme indicação do Ministério da } \\
\text { Saúde, pois este é o principal fator de } \\
\text { identificação para o tratamento precoce } \\
\text { e o melhor prognósticos de cura para o } \\
\text { câncer de colo uterino. }\end{array}$ \\
\hline 10 & $\begin{array}{l}\text { Google } \\
\text { Acadêmico }\end{array}$ & $\begin{array}{l}\text { Atuação da enfermeira } \\
\text { frente aos fatores que } \\
\text { interferem na adesão de } \\
\text { mulheres idosas ao } \\
\text { exame de Papanicolau }\end{array}$ & $\begin{array}{l}\text { Oliveira, D. da S., } \\
\text { Sá, A. V. V., } \\
\text { Gramacho, R. de } \\
\text { C. C. V., Silva, R. } \\
\text { de C. V. da, \& } \\
\text { Oliveira, J. de S. } \\
\text { (2019) }\end{array}$ & $\begin{array}{l}\text { Discutir a atuação da } \\
\text { enfermeira frente aos } \\
\text { fatores que interferem na } \\
\text { adesão de mulheres idosas } \\
\text { ao exame de Papanicolau. }\end{array}$ & $\begin{array}{l}\text { A importância da atuação da enfermeira } \\
\text { para identificar os fatores que impedem } \\
\text { a adesão das idosas ao rastreamento do } \\
\text { câncer do colo do útero através do } \\
\text { exame de Papanicolau. }\end{array}$ \\
\hline 11 & $\begin{array}{l}\text { Google } \\
\text { Acadêmico }\end{array}$ & $\begin{array}{ll}\begin{array}{l}\text { Experiência } \\
\text { enfermeira }\end{array} & \text { da } \\
\text { prevenção do câncer } \\
\text { cérvico-uterino }\end{array}$ & $\begin{array}{l}\text { Dantas, P. V. J., } \\
\text { Leite, K. N. S., } \\
\text { César, E. S. R., da } \\
\text { Costa, S., Silva, R., } \\
\text { de Souza, T. A., \& } \\
\text { do Nascimento, B. } \\
\text { B. (2018) }\end{array}$ & $\begin{array}{l}\text { Descrever a experiência } \\
\text { da enfermeira, ao realizar } \\
\text { a consulta de enfermagem, } \\
\text { na prevenção do câncer do } \\
\text { colo uterino, segundo os } \\
\text { preceitos da Teoria de } \\
\text { Enfermagem Humanística }\end{array}$ & $\begin{array}{l}\text { A enfermagem necessita aplicar todos } \\
\text { os instrumentos de comunicação } \\
\text { disponíveis para efetivar o diálogo } \\
\text { preciso para um a consulta com enfoque } \\
\text { na atenção integral da mulher, tendo em } \\
\text { vista que a consulta de enfermagem, } \\
\text { acima de tudo, constitui um espaço de } \\
\text { educação em saúde, em que o usuário é } \\
\text { convidado a protagonizar tais } \\
\text { momentos. Assim, em conformidade } \\
\text { com o princípio da integralidade, a } \\
\text { abordagem do profissional de saúde não } \\
\text { se deve restringir à assistência cur ativa, } \\
\text { e sim buscar dimensionar fatores de } \\
\text { risco à saúde e, por conseguinte, a } \\
\text { execução de açães preventivas e de } \\
\text { promoção, a exemplo da educação em } \\
\text { saúde. }\end{array}$ \\
\hline 12 & $\begin{array}{l}\text { Google } \\
\text { Acadêmico }\end{array}$ & $\begin{array}{l}\text { O papel do enfermeiro } \\
\text { na prevenção do } \\
\text { câncer de colo do útero } \\
\text { em unidade básica de } \\
\text { saúde enfatizando o } \\
\text { acolhimento }\end{array}$ & 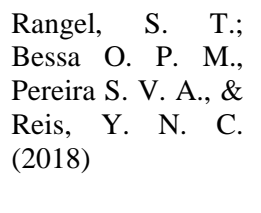 & 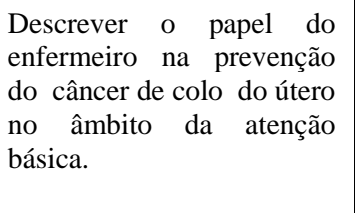 & $\begin{array}{l}\text { O enfermeiro deverá propor-se a } \\
\text { desenvolver um clima de confiança, } \\
\text { afiançando um atendimento } \\
\text { humanizado, com preparo técnico e } \\
\text { sensibilidade para cooperar na } \\
\text { qualidade do atendimento }\end{array}$ \\
\hline
\end{tabular}

Fonte: Autores (2021).

O material retro mencionado foi utilizado como base de apoio para a confecção do presente trabalho, vez que tratava de maneira clara sobre o tema, sem extrapolar as delimitações anteriormente realizadas. Cabe ressaltar que o material é de grande relevância para o contexto social, vez que amplamente informativos, não possuindo caráter exclusivo de cunho técnico.

\section{Conclusão}

Após as leituras realizadas para a confecção do presente trabalho restou clara a importância do enfermeiro na prevenção do câncer de colo de útero por meio de ações de promoção, prevenção e assistência à saúde das mulheres que tenham iniciado a vida sexual. Bem como restou evidente a importância do diagnostico precoce para a redução da alta mortalidade do CCU e, ainda, a necessidade de informações claras sobre o exame de papanicolau e a quebra dos estigmas que impedem mais de um milhão de mulheres de realizá-lo.

O enfermeiro exerce, também, o papel de educador, tendo em vista sua influência através da comunicação, ele constitui um vínculo com a paciente fazendo com que ela possa expor seus problemas e estilo de vida, onde o enfermeiro consiga identificar qual atendimento a paciente necessita (Silva et al., 2013). 
A atuação no enfermeiro frente à mulher portadora de câncer de colo de útero é grande relevância, vez que esse possui diversas funções, seja como agente de saúde, seja na participação junto à sociedade, ou ainda, na fonte de informações cientificas que possui para sanar todos as duvidas do exame a ser realizado, bem como sobre a doença e sua gravidade.

De tal modo, considerando o avanço contínuo da ciência, torna-se imprescindível a continuação de pesquisas no ramo, a fim de que o CCU possa ser diagnosticado o mais precocemente possível, conferindo a mulher portadora da patologia maiores chances de cura, bem como ser realizadas maiores campanhas de prevenção e conscientização das mulheres em idade de risco para que acolham o exame do papanicolau como exame de rotina.

\section{Referências}

Brasil. (2004). Ministério da Saúde. Secretaria de Atenção à Saúde. Departamento de Ações Programáticas Estratégicas. Política Nacional de Atenção Integral à Saúde da Mulher - Princípios e Diretrizes. Série C. Projetos, Programas e Relatórios. http://bvsms.saude.gov.br/bvs/publicacoes/politica_nac_atencao_mulher.pdf

Brasil. (2002). Ministério da Saúde. Secretaria de Atenção à Saúde. Manual Técnico. Profisionais de Saúde. http://bvsms.saude.gov.br/bvs/publicacoes/inca/manua_assistencia.pdf

Brasil. (2020). Instituto Nacional do Câncer. Incidência do Câncer no Brasil. Estimativa 2020. Ministério da Saúde destinadas à Saúde da Mulher. http://portalms.saude.gov.br/saude-para-voce/saude-da-mulher/publicacoes.

Alves, R. S. S., Sousa, F. L. L., Leite, A. C., Silva, M. P. B., Silva, 1. 1., Silva, J. M., Silva, 1. A. C., Martins, I. M., Fonseca, R. M., Silva, 1. C., Medeiros, G. F., Sampaio, B. C. A. B., Santos, J. F., Souza, R. D. \& Araújo, 1. V. F. (2021). Women's health: Preventive measures for cervical cancer. Research, Society and Development. 10(1), e32610110503. DOI: 10.33448/rsd-v10i1.10503. https://rsdjournal.org/index.php/rsd/article/view/10503

Barbosa, G. S. L., Silva Souza, A. T., Júnior, F. C. F. V., Júnior, E. J. F., de Melo Oliveira, D. M., Martins, F. L. R., \& dos Santos Pedrosa, J. I. (2020). Realização do exame citopatológico em mulheres: uma revisão integrativa. Research, Society and Development, 9(11).

Valente, C. A, Andrade, C. A., Oliveira, M. B. O. \& Soares, S. R. S. (2009). Conhecimento de Mulheres Universitárias em Relação à Importância do Exame Citopatológico de Papanicolaou. Rev. esc. enferm. USP 43(spe2). https://doi.org/10.1590/S0080-62342009000600008

Carneiro, C. P. F., Pereira, D. M., Pereira, A. T \& et al. (2019). O papel do enfermeiro frente ao câncer de colo uterino. Revista Eletrônica Acervo Saúde. .35(1), ed 1362. P. 1-9. DOI: https://doi.org/10.25248/reas.e1362.2019. https://acervomais.com.br/index.php/saude/article/view/1362/924

Cruz, L. M. B., \& Loureiro, R. P. (2008). A comunicação na abordagem preventiva do câncer do colo do útero: importância das influências histórico-culturais e da sexualidade feminina na adesão às campanhas. Saúde e Sociedade. http://www.scielo.br/scielo.php?script=sci_arttext\&pid=\$010412902008000200012

Conceição, J. P. S., Medeiros, M. M. S., Rodrigues, L. M. S., Braz, M. R., Balbino, C. M. \& Silvino, Z. R. (2017). O conhecimento do enfermeiro sobre a prevenção do câncer de colo de útero na atenção básica. Revista Enfermagem Atual In Derme. https://doi.org/10.31011/reaid-2017-v.2017-n.0-art.552. http://www.revistaenfermagematual.com.br/index.php/revista/article/view/552/523

Dantas, P. V. J., Leite, K. N. S., César, E. S. R., da Costa, S., Silva, R., de Souza, T. A., \& do Nascimento, B. B. (2018). Conhecimento das mulheres e fatores da não adesão acerca do exame papanicolau. Revista de Enfermagem UFPE Online. 12(3).

Leite, A. C., Silva, M. P. B., Alves, R. S. S., Feitosa, L. M. H., Ribeiro, R., Prado, A. M., \& Soares, N. C. F. B. (2020). Atribuições do enfermeiro no rastreamento do câncer de colo do útero em pacientes atendidas na Unidade Básica de Saúde. Research, Society and Development, 9(11).

Eduardo, K. G. T. \& Sousa, I. G. S. \& Moura, E. R. F. (2008). Prevenção do câncer de colo uterino: percepções de mulheres ao primeiro exame e atitudes profissionais. Rev. RENE. 9(2), 38-46. http://www.repositorio.ufc.br/bitstream/riufc/4394/1/2008_art_kgteduardo.pdf.

Melo S.C.C. S., Prates L, \& Carvalho M. D. B. (2009). Alterações citopatológicas e fatores de risco para ocorrência do câncer de colo uterino. Revista gaúcha de enfermagem. 30 (4).

Moura, A. D. A.. Conhecimento e motivações das mulheres acerca do exame de papanicolau: subsídios para a para a prática de enfermagem. REV. RENE. 11(1) HTTP://REPOSITORIO.UFC.BR/BITSTREAM/RIUFC/4013/1/2010_ART_ADAMOURA.PDF.

Oliveira i. S. B., Panobianco M. S., Pimentel a. V., Nascimento 1. C., \& Gozzo T. O. (2010). Ações das equipes de saúde da família na prevenção e controle do câncer de colo de útero. Cienc cuid saude; 9(2): 220-7.

Oliveira, m. M., pinto, i. C., \& coimbra, v. C. C. (2017). Potencialidades no atendimento integral: a prevenção do câncer do colo do útero na concepção de usuárias da estratégia saúde da família. Rev. Latino-am. Enfermagem, 15(3), p. 426-430. Http://www.scielo.br/scielo.php?pid=s010411692007000300010\&script=sci_arttext\& tlng=pt.

Pereira, a. S., Shitsuka, D. M., Parreira, F. J., \& Shitsuka, R. (2018). Metodologia da pesquisa científica.[e-book]. Santa maria. Ed. Uab/nte/ufsm. Https://repositorio. Ufsm. Br/bitstream/handle/1/15824/lic_computacao_metodologia-pesquisa-cientifica. Pdf.

Rangel, S. T.; Bessa O. P. M., Pereira S. V. A., \& Reis, Y. N. C. (2018). O papel do enfermeiro na prevenção do câncer de colo do útero em unidade básica de saúde enfatizando o acolhimento. Múltiplos acessos, 3(1).

Ricci, S. S. (2008). Enfermagem Materno Infantil - Neonatal e Saúde da Mulher. Ed. Guanabara Koogan. 
Research, Society and Development, v. 10, n. 13, e137101321040, 2021

(CC BY 4.0) | ISSN 2525-3409 | DOI: http://dx.doi.org/10.33448/rsd-v10i13.21040

Siqueira, G. S. (2014) Citopatologia Como Prevenção do Câncer do Colo Uterino. Cadernos de Graduação - Ciências Biológicas e da Saúde Unit. https://periodicos.set.edu.br/index.php/cadernobiologicas/article/view/1179.

Silva, M. L., Nunes, J. S. S., Oliveira, K. S., \& Leite, T. A. S.. (2020). O Conhecimento de Mulheres Sobre o Câncer de Colo de Útero: Uma Revisão Interativa. Brazilian Jounal of Healt Review. Vitória da Conquista, 3 (4), p. $7263-1275$. https://www.brazilianjournals.com/index.php/BJHR/article/view/12566/0.

Silva, S. M. S. (2013) Atuação do enfermeiro de PSF no processo da realização do papanicolaou. Congresso Brasileiro de Medicina Família e Comunidade. $12(405)$.

Silva O., Sá, A. V., Gramacho, R. D. C. C. V., da Silva, R. D. C. V., \& de Souza Oliveira, J. (2019). Atuação da enfermeira frente aos fatores que interferem na adesão de mulheres idosas ao exame de Papanicolau. Revista Enfermagem Contemporânea, 8(1), 87-93.

Soares M. C., Mishima S. M., Silva R. C., Ribeiro C. V., Meincke S. M. K., \& Corrêa A. C. L. (2011) Câncer de colo uterino: atenção integral à mulher nos serviços de saúde. Revista Gaúcha de Enfermagem. 32(3): 502-8.

Sobotta. (2012) Atlas de Anatomia Humana: Órgãos Internos, Ed.: Guanabara Koogan. 